\title{
Generalized Master Equations Leading to Completely Positive Dynamics
}

\author{
Bassano Vacchini \\ Dipartimento di Fisica, Università degli Studi di Milano, Via Celoria 16, I-20133 Milan, Italy \\ and INFN, Sezione di Milano, Via Celoria 16, I-20133 Milan, Italy
}

(Received 16 July 2016; published 30 November 2016)

\begin{abstract}
We provide a general construction of quantum generalized master equations with a memory kernel leading to well-defined, that is, completely positive and trace-preserving, time evolutions. The approach builds on an operator generalization of memory kernels appearing in the description of non-Markovian classical processes and puts into evidence the nonuniqueness of the relationship arising due to the typical quantum issue of operator ordering. The approach provides a physical interpretation of the structure of the kernels, and its connection with the classical viewpoint allows for a trajectory description of the dynamics. Previous apparently unrelated results are now connected in a unified framework, which further allows us to phenomenologically construct a large class of non-Markovian evolutions taking as the starting point collections of time-dependent maps and instantaneous transformations describing the microscopic interaction dynamics.
\end{abstract}

DOI: 10.1103/PhysRevLett.117.230401

In the presence of an external environment, the time evolution of a quantum system is no more given by a reversible unitary dynamics. For the description of such open quantum systems, one of the important issues is the determination of equations providing a well-defined reduced dynamics [1]. While for a reversible quantum evolution Stone's theorem implies that the determination of the time evolution amounts to the identification of the system Hamiltonian, no such general result is available for a generic reduced dynamics. Such a result would be of major importance also in view of phenomenological approaches, since the very complexity of a general system environment setting suggests that a microscopic approach starting from a Hamiltonian description for both the system and the environment is often unfeasible. Indeed, while perturbative techniques are known in order to formally obtain the reduced dynamics of the system degrees of freedom in the form both of integro-differential equations and of time-local master equations [1], the perturbative analysis is quite cumbersome, and, in particular, preservation of complete positivity $(C P)$ is not warranted unless all terms of the perturbation expansion are considered. The property of $C P$ [2] ensures positivity of the time evolution in the presence of an arbitrary ancillary system regardless of its interaction with the system of interest. Given a factorized initial system-environment state and a unitary interaction between the system and the environment, the reduced time evolution has to be $C P$ [3]. It is therefore natural to ask phenomenological evolution equations to preserve this property. A key characterization has been given for the case in which the evolution maps combine as $\Phi(t+s)=\Phi(t) \Phi(s)$ for positive times only, corresponding to a semigroup composition law. The most general expression for a semigroup of quantum $C P$ transformations is given by $\Phi(t)=e^{\mathcal{L} t}$, where the so-called Lindblad generator $\mathcal{L}$ solves the master equation $\dot{\rho}(t)=\mathcal{L} \rho(t)$, and its structure is fixed by a famous theorem [4]. Further important results on the possible structure of time-local master equations leading to a well-defined $C P$ dynamics have been obtained. In such a case, Hermiticity and trace preservation already strongly constrain the operator structure of the equation, and this has allowed us to determine quite general sufficient conditions warranting the existence of a $C P$ reduced dynamics [5]. Basically, one considers a master equation whose operator structure is the same as in the semigroup case, but coefficients and operators can now depend on time, and one looks for conditions on this time dependence warranting $C P$. Much less is known in the case of generalized master equations of the form

$$
\frac{d}{d t} \rho(t)=\int_{0}^{t} d \tau \mathcal{K}(t-\tau) \rho(\tau),
$$

where the operator $\mathcal{K}(t)$ is called a memory kernel (MK), possibly including on the right-hand side a term of the form $\mathcal{I}(t) \rho(0)$, that is, an inhomogeneous contribution. In this framework, even the requirement of Hermiticity and trace preservation is not easily satisfied, let alone $C P$. Moreover, in this case one often lacks a simple connection between the expression of the MK and the basic microscopic physical interaction mechanisms, at variance with the time-local case, in which coefficients can often naturally be interpreted as rates and the so-called Lindblad operators appearing in the structure can typically be connected, e.g., with transitions among system states. This fact further hindered the determination of a well-defined MK on the basis of physical intuition, and indeed innocent-looking or apparently physically motivated MKs actually lead to ill-defined time evolutions [6]. Despite this, well-defined generalized master equations have been obtained, both within mathematical or phenomenological approaches [7-14] and considering definite microscopic models [15-17]. However, a general construction both encompassing known examples and 
providing hints for the determination of generalized master equations based on the introduction of quantum maps, to be guessed phenomenologically or determined from microscopic physical interactions, is yet not available. The determination of time evolutions beyond a semigroup law is also relevant in order to describe quantum memory effects [18]. In this Letter, we show how to obtain general classes of quantum MK master equations building on the structure of a classical MK leading to non-Markovian classical processes. In moving from the classical to the quantum realm, the correspondence between classical quantities and quantum operators is not unique, and the different viable options of operator ordering lead to a rich structure, reflecting themselves in the different time ordering of the operators in the solution. Indeed this subtle issue allows us to understand and connect apparently unrelated results. Coming from classical non-Markovian processes, also a trajectory viewpoint is naturally available, allowing a physical interpretation of the operators determining the MK. Our result further shows how much can be learned coming to quantum mechanics from a classical probabilistic viewpoint [3].

Classical and quantum memory kernel. - Let us consider a classical system living on a denumerable set of states. Once in a state $k$, it will remain there for a time determined by a probability distribution $f_{k}(t)$, called the waiting time distribution, and then jump to another state $n$ with a probability given by the element $\pi_{n k}$ of a given stochastic matrix. To each waiting time distribution $f_{k}(t)$ is associated its survival probability $g_{k}(t)=1-\int_{0}^{t} d \tau f_{k}(\tau)$, providing the probability not to leave the state up to time $t$. The conditional transition probability of the process $T_{n m}(t)$, namely, the probability to be in state $n$ at time $t$ under the condition of starting in $m$ at time zero, obeys the integrodifferential equation [19]

$$
T_{n m}(t)=\delta_{n m} g_{m}(t)+\int_{0}^{t} d \tau \sum_{k} w_{n k}(t-\tau) T_{k m}(\tau),
$$

where the function $w_{n k}(t)$ has a simple expression in the Laplace transform, namely, $\hat{w}_{n k}(u)=\hat{g}_{n}(u) \pi_{n k} \hat{f}_{k}(u) / \hat{g}_{k}(u)$. The case of a Markovian process is then recovered for waiting time distributions of exponential form with rate $\lambda_{k}$, corresponding to $w_{n k}(t)=e^{-\lambda_{n}(t-\tau)} \pi_{n k} \lambda_{k}$. Considering a process starting in a fixed state, as described in Supplemental Material [20], its probability vector $P_{n}(t)$ obeys the same generalized master equation, which in the Laplace transform reads

$$
\begin{aligned}
u \hat{P}_{n}(u)-P_{n}(0)= & \sum_{m}\left[\pi_{n m} \frac{\hat{f}_{m}(u)}{\hat{g}_{m}(u)}\right. \\
& \left.-\delta_{n m}\left(\frac{1}{\hat{g}_{m}(u)}-u\right)\right] \hat{P}_{m}(u),
\end{aligned}
$$

providing a convenient starting point for a quantum generalization. Indeed, written in this way, the MK is determined by quantities, such as stochastic matrix and waiting time distribution, admitting a direct physical interpretation. It further warrants that the solution $P_{n}(t)$ is at any time a well-defined probability vector and arises from a reading of the time evolution in terms of trajectories, corresponding, in particular, to examples of so-called semiMarkov processes [23]. At variance with Ref. [10], where the existence of such processes was a motivation to look for quantum MKs in the form of time-dependent Lindblad generators, further pointing to conditions on the warranting of $C P$ based on a perturbative analysis of the solution, we will here more closely focus on the specific form of the MK appearing in Eq. (3), thus, in particular, keeping the connection with a trajectory viewpoint. This aspect was partially developed in Ref. [13], though fully missing the deep connection with the MK of classical non-Markovian processes and inadvertently using a particular operator ordering.

In quantum mechanics, probability vectors are replaced by statistical operators, and, in order to obtain a suitable MK for Eq. (1), one can start from (3) replacing the different $\mathbb{C}$-number quantities by operator-valued ones according to

$$
\hat{\mathcal{K}}(u)=\mathcal{O}[\pi[\hat{f}(u) / \hat{g}(u)]]-\mathcal{O}\left[\hat{g} \mathcal{G}(u)^{-1}-u\right],
$$

where $\mathcal{O}[\cdot]$ denotes an operator replacement rule also taking into account the issue of operator ordering. This ordering will determine the distribution of the action in time of the different noncommuting operators. Note that, while the dynamics will be defined in terms of time-dependent operators, it is convenient for the sake of simplicity to introduce the replacement rule in the Laplace transform. The quantum counterpart of the stochastic matrix $\pi$ is an arbitrary $C P$ trace-preserving transformation $\mathcal{E}$, while the waiting time distribution $f(t)$ will be replaced by $f(t) \mathcal{F}(t)$, with $\mathcal{F}(t)$ a collection of time-dependent $C P$ tracepreserving maps describing the transformation of the system between jumps. Similarly, the survival probability $g(t)$ goes over to $g(t) \mathcal{G}(t)$, where again the maps $\mathcal{G}(t)$ are $C P$ trace-preserving and such that $\mathcal{G}(0)=\mathbb{1}$. We then consider the following operator replacement rule:

$$
\mathcal{O}[\pi[\hat{f}(u) / \hat{g}(u)]] \rightarrow \hat{g \mathcal{G}}(u)^{-1} \hat{f \mathcal{F}}(u) \mathcal{E},
$$

where $\hat{f} \mathcal{F}(u)$ denotes the Laplace transform of $f(t) \mathcal{F}(t)$ and similarly for $g(t) \mathcal{G}(t)$, leading to

$$
\hat{\mathcal{K}}_{R}(u)=\hat{g \mathcal{G}}(u)^{-1} \hat{f} \mathcal{F}(u) \mathcal{E}-\left[\hat{g \mathcal{G}}(u)^{-1}-u\right]
$$

where note that operator ordering plays a role only in the first term on the right-hand side of Eq. (4). This operator MK immediately leads to the expression of the time evolution map [20] transforming the initial quantum state in the time-evolved one: 


$$
\hat{\Phi}_{R}(u)=[\mathbb{1}-\hat{f \mathcal{F}}(u) \mathcal{E}]^{-1} \hat{g} \mathcal{G}(u),
$$

so that in the time domain, replacing the inverse by a Neumann series and exploiting the fact that multiplication goes over to convolution, we obtain for $\rho(t)=\Phi_{R}(t) \rho(0)$

$$
\rho(t)=\sum_{n=0}^{\infty}\left[*^{n}(f \mathcal{F} \mathcal{E}) *(g \mathcal{G})\right](t) \rho(0),
$$

where $*^{n}$ denotes the $n$-fold convolution. This very expression warrants $C P$ of $\Phi_{R}(t)$, as composition of $C P$ maps, while the requirement of trace preservation, calling for a kind of balance between the two contributions of Eq. (6), can be read directly from the kernel as shown in Ref. [20] and leads to

$$
d \operatorname{Tr}\{g(t) \mathcal{G}(t) \rho\} / d t=-\operatorname{Tr}\{M(t) \rho\},
$$

where $\hat{M}(u)=\hat{g} \mathcal{G}(u)^{-1} \hat{f \mathcal{F}}(u) \hat{\mathcal{E}} \hat{\mathcal{G}}(u)$. Since $\mathcal{F}(t)$ and $\mathcal{G}(t)$ are trace-preserving, (9) takes the simple form

$$
d g(t) / d t=-f(t)
$$

namely, just the basic relation between an arbitrary waiting time distribution $f(t)$ and its survival probability $g(t)$. We have thus obtained in a straightforward way a class of MK ensuring $C P$ and trace preservation of the associated time evolution, both nontrivial requirements in the case of integro-differential equations. It immediately appears that due to the noncommutativity of operators in quantum mechanics besides (5), for the very same collection of time-dependent maps, one can also consider a different operator replacement:

$$
\mathcal{O}[\pi[\hat{f}(u) / \hat{g}(u)]] \rightarrow \mathcal{E} f \hat{\mathcal{F}}(u) \hat{g} \mathcal{G}(u)^{-1},
$$

identifying a different kernel $\mathcal{K}_{L}(t)$ and an evolution map $\Phi_{L}(t)$ leading for $\rho(t)=\Phi_{L}(t) \rho(0)$ to

$$
\rho(t)=\sum_{n=0}^{\infty}\left[(g \mathcal{G}) *^{n}(\mathcal{E} f \mathcal{F})\right](t) \rho(0) .
$$

Again, one immediately has $C P$, while the trace preservation condition takes the simpler form $d \operatorname{Tr}\{g(t) \mathcal{G}(t) \rho\} /$ $d t=-\operatorname{Tr}\{\mathcal{E} f(t) \mathcal{F}(t) \rho\}$, still satisfied thanks to the trace preservation of the single contributions and Eq. (10). Kernels falling within this latter choice have been obtained in Ref. [14]. The two MK thus obtained, arising from different operator orderings, indeed lead to different dynamical evolution equations. While in the Markovian case the structure of quantum dynamical semigroups as the quantum counterpart of classical Markov semigroups appears to be uniquely fixed and captured by the expression of the Lindblad generator [4], for the quantum counterpart of non-Markovian classical processes a greater freedom appears. In a Lindblad master equation, we only have to fix the Lindblad operators; here the presence of a MK implies that also the time sequence in the action of the different operators is relevant. We stress moreover that even for a fixed MK Eq. (1) can be written in different ways. Indeed, while the expressions of the MK in the Laplace transform are quite simple, in the time domain it is convenient to introduce also an inhomogeneous term, so that the generalized master equations corresponding to the kernels $\mathcal{K}_{R, L}(t)$ read [20]

$$
\begin{aligned}
\frac{d}{d t} \rho(t) & =\int_{0}^{t} d \tau \mathcal{K}_{R, L}(t-\tau) \rho(\tau) \\
& =\int_{0}^{t} d \tau \mathcal{W}_{R, L}(t-\tau) \rho(\tau)+\mathcal{I}(t) \rho(0),
\end{aligned}
$$

where the common inhomogeneous term reads $\mathcal{I}(t)=$ $d[g(t) \mathcal{G}(t)] / d t$, while the kernels $\mathcal{W}_{R, L}$ are given by

$$
\begin{gathered}
\mathcal{W}_{R}(t)=d[f(t) \mathcal{F}(t)] / d t \mathcal{E}+\delta(t) f(0) \mathcal{F}(0) \mathcal{E}, \\
\mathcal{W}_{L}(t)=d[H(t)] / d t
\end{gathered}
$$

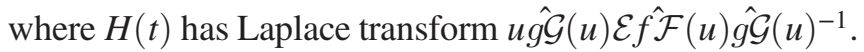
Note the different complexity in the kernels, which does not always allow for a direct interpretation in terms of the relevant collections of $C P$ maps determining the dynamics. The difference between $\mathcal{W}_{R}$ and $\mathcal{W}_{L}$ just arises due to noncommutativity, even though this simple connection is transparent only in the Laplace domain. While further choices can be considered moving $\mathcal{E}$ in different positions [20], the one considered here is suggested by the trajectory expansion (18).

An interesting case arises assuming as an ansatz that the dynamics between the microscopic interaction events, described by the map $\mathcal{E}$, is given by a quantum dynamical semigroup with generator $\mathcal{L}$ in Lindblad form. In this case, the only relevant ordering depends on the positioning of $\mathcal{E}$ with respect to the functions of $\mathcal{L}$. We can thus consider the replacement

$$
\mathcal{O}[\pi[\hat{f}(u) / \hat{g}(u)]]=\frac{\hat{f}(u-\mathcal{L})}{\hat{g}(u-\mathcal{L})} \mathcal{E},
$$

leading to the master equation [20]

$$
\frac{d}{d t} \rho(t)=\mathcal{L} \rho(t)+\int_{0}^{t} d \tau e^{\mathcal{L}(t-\tau)} k(t-\tau) \mathcal{M} \rho(\tau),
$$

where the function $k(t)$ is given in the Laplace transform by $\hat{k}(u)=\hat{f}(u) / \hat{g}(u)$, in analogy with the classical MK in (3), and $\mathcal{M}=(\mathcal{E}-\mathbb{1})$ is itself a generator in Lindblad form. The alternative choice of operator ordering in (16) leads 
to a similar equation where the position of $\mathcal{L}$ and $\mathcal{M}$ is exchanged.

Trajectory description and physical examples.-We now want to connect the obtained results more closely to a description of the dynamics in terms of trajectories, further pointing to physical realizations. Let us first observe that the time evolution maps $\Phi_{R}(t)$ and $\Phi_{L}(t)$ admit the representations

$$
\begin{aligned}
\Phi_{R}(t)= & p_{R}^{0}(t) \mathcal{G}(t)+\sum_{n=1}^{\infty} \int_{0}^{t} d t_{n} \ldots \int_{0}^{t_{2}} d t_{1} \\
& \times p_{R}^{n}\left(t ; t_{n}, \ldots, t_{1}\right) \mathcal{F}\left(t-t_{n}\right) \mathcal{E} \ldots \mathcal{F}\left(t_{2}-t_{1}\right) \mathcal{E} \mathcal{G}\left(t_{1}\right),
\end{aligned}
$$

$$
\begin{aligned}
\Phi_{L}(t)= & p_{L}^{0}(t) \mathcal{G}(t)+\sum_{n=1}^{\infty} \int_{0}^{t} d t_{n} \ldots \int_{0}^{t_{2}} d t_{1} \\
& \times p_{L}^{n}\left(t ; t_{n}, \ldots, t_{1}\right) \mathcal{G}\left(t-t_{n}\right) \ldots \mathcal{E F}\left(t_{2}-t_{1}\right) \mathcal{E F}\left(t_{1}\right)
\end{aligned}
$$

with $p_{R, L}^{n}\left(t ; t_{n}, \ldots, t_{1}\right)$ the exclusive probability densities for jumps corresponding to the action of $\mathcal{E}$ at times $t_{1}, \ldots, t_{n}$ within the time interval from 0 to $t$. They are given by

$$
\begin{aligned}
& p_{R}^{n}\left(t ; t_{n}, \ldots, t_{1}\right)=f\left(t-t_{n}\right) \ldots f\left(t_{2}-t_{1}\right) g\left(t_{1}\right), \\
& p_{L}^{n}\left(t ; t_{n}, \ldots, t_{1}\right)=g\left(t-t_{n}\right) \ldots f\left(t_{2}-t_{1}\right) f\left(t_{1}\right),
\end{aligned}
$$

where the different time arguments become relevant in the integrals (18) and (19) due to a connection with the operator action. This fact embodies the further freedom available in this situation with respect to the Markovian case. In particular, (20) is the standard expression considered in a renewal process describing events randomly taking place after a time interval determined by the distribution $f(t)$. As discussed in Refs. [24,25] and detailed in Ref. [20], Eqs. (18) and (19) provide a trajectory description of the dynamics at the level of the statistical operator in that they express the solution of the master equation as a sum of contributions corresponding to statistical operators determined by the number and the time of jumps, weighted according to the probability densities (20) and (21). Each contribution is characterized by the repeated action of the map $\mathcal{E}$ at the given times, together with the application of the maps $\mathcal{F}(t)$ and $\mathcal{G}(t)$ in the intermediate time evolution, in analogy to what happens in the standard Markovian case [3].

It turns out that Eq. (18) corresponding to the kernel (6) includes and generalizes [13], allowing for possibly distinct collections $\mathcal{F}(t)$ and $\mathcal{G}(t)$. It thus provides the theoretical framework encompassing quantum collisional models [16], including a most recently introduced generalization [17], where the time evolution of the system in the first time interval is different from those in later ones. Conversely, Eq. (19) describes a situation in which the dynamics has a different characterization in the last time interval. For a semigroup evolution among jumps, as in Eq. (17), one recovers a model of non-Markovian dynamics first considered in a simplified case in Ref. [8]. If on top of this $\mathcal{E}$ acts as the identity, independently of the waiting time distribution one recovers a semigroup dynamics. Keeping a nontrivial $\mathcal{E}$ and $f(t)$ but assuming the system does not appreciably change between jumps, one obtains models of a so-called continuous time quantum random walk [26,27].

The situation described by Eq. (19) instead, arising in the presence of the kernel $\mathcal{K}_{L}(t)$ determined by Eq. (11), for the case of an intermediate semigroup time evolution encompasses the description of non-Markovian dynamics in the physics of the micromaser $[15,28,29]$. The micromaser or one-atom maser provides one of the most fundamental systems to study light-matter interaction [30]. In this system, single two-level atoms are sent through a resonant high-quality single-mode microwave cavity. The interaction between the single atoms and the cavity mode is described by a Jaynes-Cummings Hamiltonian and takes place for the time the atom takes to cross the cavity, assumed to be constant. By taking the trace with respect to the atom degrees of freedom of this unitary interaction, one obtains a $C P$ trace-preserving transformation $\mathcal{E}$. In between the arrival of subsequent atoms, the cavity mode dynamics is well described by a semigroup evolution with a standard Lindblad generator $\mathcal{L}$ giving the Markovian decay of the cavity field. The further information necessary in order to determine the dynamics is the distribution in time of the atoms flying through the cavity. For the case of a Poissonian distribution of the time of arrivals of atoms, the dynamics of the field can be described by a Markovian master equation, as can be seen considering an exponential waiting time distribution and assuming the semigroup assignment $\mathcal{F}(t)=\mathcal{G}(t)=e^{\mathcal{L} t}$ in Eq. (19). Different distributions, allowing for non-Markovian effects, call for a more general treatment and lead to MK master equations. Note that Eq. (19) can actually encompass more general situations with respect to an intermediate semigroup evolution. Our approach thus recovers on the one side quantum collisional models, showing that they can be generalized to include general waiting time distributions still leading to closed evolution equations, and on the other side a dynamics like the one of the micromaser, pointing to the fact that it can be extended to consider situations in which the intermediate time evolution is not necessarily of the semigroup type. In particular, it shows a common path to describe the two phenomena.

We stress that the obtained results are not restricted to the case of a finite-dimensional Hilbert space; indeed, while quantum collisional models have been realized up to now considering qubit systems, in the case of the micromaser one is actually interested in how the field dynamics is affected by the atoms passing through the cavity. While the expansion of the time evolution in terms of trajectories as in 
Eq. (19) is easily obtained in this approach from the MK (6), more general situations can be obtained starting directly from Eq. (21) and considering rather than an ordinary a so-called modified renewal process, in which the first waiting time is different from the remaining ones and described by a distribution $f_{1}(t)$. In this case, the evolution map is given by

$$
\begin{aligned}
\Phi(t)= & g_{1}(t) e^{\mathcal{L} t}+\sum_{n=1}^{\infty} \int_{0}^{t} d t_{n} \ldots \int_{0}^{t_{2}} d t_{1} \\
& \times g\left(t-t_{n}\right) e^{\mathcal{L}\left(t-t_{n}\right)} \ldots \mathcal{E} f\left(t_{2}-t_{1}\right) \\
& \times e^{\mathcal{L}\left(t_{2}-t_{1}\right)} \mathcal{E} f_{1}\left(t_{1}\right) e^{\mathcal{L} t_{1}}
\end{aligned}
$$

with Lindblad generator $\mathcal{L}$ and jump map $\mathcal{E}$ as described above. As detailed in Ref. [20], one still obtains a closed evolution equation in integro-differential form as in Eq. (1) with kernel

$$
\begin{aligned}
\hat{\mathcal{K}}(u)= & \mathcal{L}+\left\{1-\mathcal{M}\left[\hat{S}(u-\mathcal{L})-\hat{S}_{1}(u-\mathcal{L})\right]\right\}^{-1} \\
& \times \mathcal{M} \hat{k}_{1}(u-\mathcal{L}) .
\end{aligned}
$$

Here $\mathcal{M}$ is defined as in (17), and the classical kernel $\hat{k}_{1}(u)=\hat{f}_{1}(u) / \hat{g}(u)$ appears operator valued due to the dependence on $\mathcal{L}$, while $S(t)$ is the so-called renewal density or sprinkling distribution [31], giving the probability for a jump to occur at a given time, defined for an ordinary process as $\hat{S}(u)=\hat{f}(u) /[1-\hat{f}(u)]$ and as $\hat{S}_{1}(u)=\hat{f}_{1}(u) /[1-\hat{f}(u)]$ for a modified one, again appearing operator valued. For the special case of a stationary distribution of jumps, one has the constraint $f_{1}(t)=g(t) /\langle\tau\rangle$, with $\langle\tau\rangle$ the mean waiting time associated to the reference distribution $f(t)$, leading to the model obtained in a much less straightforward way in Ref. [15]. Also here all terms appearing in the MK have a direct meaning as physical transformation maps or quantities related to the renewal process giving the time distribution of the jumps describing microscopic interaction events. Furthermore, despite the complicated expression of the MK, both trace preservation and $C P$ are granted from the analysis of the ensuing dynamics in terms of trajectories, for arbitrary waiting time distributions $f(t)$ and $f_{1}(t)$ and Lindblad generator $\mathcal{L}$.

We have provided a simple general construction of a quantum MK leading to well-defined reduced dynamics. The result builds on an analogy with classical nonMarkovian processes, thus allowing for a direct physical interpretation of the different contributions appearing in the MK and for a connection to a trajectory description of the dynamics. The interpretation of the different kernels is best understood in the Laplace domain and can be read in the time domain by suitably rewriting the integro-differential equation and introducing an inhomogeneous contribution. The approach provides a general way to build a MK, complying with both trace preservation and $C P$, on the basis of microscopic physical information encoded in the collection of time-dependent maps describing the time evolution in between jumps, the channel providing the instantaneous transformation, the random distribution in time of these transformations, and the related time ordering of these maps. As in standard quantum mechanics, an operator replacement rule has to be introduced, leading, at variance with the Markovian case, to a variety of quantum stochastic dynamics corresponding to a given nonMarkovian classical one. One thus obtains a large class of non-Markovian quantum dynamics including a wide range of previous results as special cases.

The work was supported by the EU QuProCS Project (Grant Agreement No. 641277) and by a UniMI H2020 Transition Grant. Motivating discussions with M. Palma, F. Ciccarello, and S. Lorenzo as well as past correspondence with J. Cresser are also gratefully acknowledged.

[1] H.-P. Breuer and F. Petruccione, The Theory of Open Quantum Systems (Oxford University Press, New York, 2002).

[2] M. Nielsen and I. Chuang, Quantum Computation and Quantum Information (Cambridge University Press, Cambridge, England, 2000).

[3] A. S. Holevo, Statistical Structure of Quantum Theory, Lect. Notes Phys. Vol. 67 (Springer, Berlin, 2001).

[4] V. Gorini, A. Kossakowski, and E. C. G. Sudarshan, J. Math. Phys. (N.Y.) 17, 821 (1976); G. Lindblad, Commun. Math. Phys. 48, 119 (1976).

[5] H.-P. Breuer, Phys. Rev. A 75, 022103 (2007); E. Andersson, J. D. Cresser, and M. J. W. Hall, J. Mod. Opt. 54, 1695 (2007); B. Vacchini, J. Phys. B 45, 154007 (2012); S. Wißmann, H.-P. Breuer, and B. Vacchini, Phys. Rev. A 92, 042108 (2015).

[6] S. M. Barnett and S. Stenholm, Phys. Rev. A 64, 033808 (2001); A. Shabani and D. A. Lidar, Phys. Rev. A 71, 020101 (2005); S. Campbell, A. Smirne, L. Mazzola, N. Lo Gullo, B. Vacchini, Th. Busch, and M. Paternostro, Phys. Rev. A 85, 032120 (2012).

[7] S. Daffer, K. Wódkiewicz, J. D. Cresser, and J. K. McIver, Phys. Rev. A 70, 010304 (2004).

[8] A. A. Budini, Phys. Rev. A 69, 042107 (2004).

[9] A. A. Budini, Phys. Rev. E 72, 056106 (2005).

[10] H.-P. Breuer and B. Vacchini, Phys. Rev. Lett. 101, 140402 (2008).

[11] J. Wilkie and Y. M. Wong, J. Phys. A 42, 015006 (2009).

[12] A. A. Budini, Phys. Rev. A 88, 012124 (2013).

[13] B. Vacchini, Phys. Rev. A 87, 030101(R) (2013).

[14] D. Chruscinski and A. Kossakowski, Phys. Rev. A 94, 020103(R) (2016).

[15] J. D. Cresser and S. M. Pickles, J. Opt. B 8, 73 (1996).

[16] V. Giovannetti and G. M. Palma, Phys. Rev. Lett. 108, 040401 (2012); J. Phys. B 45, 154003 (2012); F. Ciccarello, G. M. Palma, and V. Giovannetti, Phys. Rev. A 87, 040103 (2013); F. Ciccarello and V. Giovannetti, Phys. Scr. T153, 014010 (2013). 
[17] S. Lorenzo, F. Ciccarello, and G. M. Palma, Phys. Rev. A 93, 052111 (2016).

[18] A. Rivas, S. F. Huelga, and M. B. Plenio, Rep. Prog. Phys. 77, 094001 (2014); H.-P. Breuer, E.-M. Laine, J. Piilo, and B. Vacchini, Rev. Mod. Phys. 88, 021002 (2016).

[19] W. Feller, Proc. Natl. Acad. Sci. U.S.A. 51, 653 (1964); D. T. Gillespie, Phys. Lett. A 64, 22 (1977); H.-P. Breuer and B. Vacchini, Phys. Rev. E 79, 041147 (2009).

[20] See Supplemental Material at for http://link.aps.org/ supplemental/10.1103/PhysRevLett.117.230401, for technical details on the derivation of equations, which incudes Refs. [21,22].

[21] H. Carmichael, An Open Systems Approach to Quantum Optics (Springer, Berlin, 1993).

[22] A. Barchielli and M. Gregoratti, Quantum Trajectories and Measurements in Continuous Time, Lect. Notes Phys. Vol. 782 (Springer, Berlin, 2009); A. Barchielli, in Stochastic Evolution of Quantum States in Open Systems and in Measurement Processes, edited by L. Diósi and B. Lukàcs (World Scientific, Singapore, 1994), pp. 1-14.
[23] D. R. Cox and H. D. Miller, The Theory of Stochastic Processes (Wiley, New York, 1965).

[24] A. A. Budini, Phys. Rev. A 88, 032115 (2013).

[25] B. Vacchini, Int. J. Quantum. Inform. 12, 1461011 (2014).

[26] E. W. Montroll and G. H. Weiss, J. Math. Phys. (N.Y.) 6, 167 (1965).

[27] M. Esposito and K. Lindenberg, Phys. Rev. E 77, 051119 (2008).

[28] J. D. Cresser, Phys. Rev. A 46, 5913 (1992).

[29] U. Herzog, Phys. Rev. A 52, 602 (1995).

[30] G. Raithel et al., in Cavity Quantum Electrodynamics, edited by P. R. Berman (Academic, San Diego, 1994), pp. 57-121; B.-G. Englert and G. Morigi, in Coherent Evolution in Noisy Environments, Lect. Notes Phys. Vol. 611, edited by A. Buchleitner and K. Hornberger (Springer, Berlin, 2002), pp. 55-106.

[31] F. Bardou et al., Lévy Statistics and Laser Cooling (Cambridge University Press, Cambridge, England, 2001). 